\title{
Introduction to the special section on rectal cancer
}

\author{
Marc J. Gollub ${ }^{1} \cdot$ Mukesh Harisinghani ${ }^{2}$
}

Published online: 13 September 2019

c) Springer Science+Business Media, LLC, part of Springer Nature 2019

Members of the Society of Abdominal Radiology (SAR), Rectal and Anal Disease Focused Panel (DFP), are honored to be presented with the opportunity to contribute articles to this Special Issue. Our members, all academic radiologists from leading medical centers in North America and Europe, enthusiastically support the incremental value of this type of specialty Panel within the Society, not only for the purpose of expanding research and education, but also to address the growing need for knowledge dissemination regarding rectal cancer, its rising incidence in the younger population and the growing use of imaging for staging and treatment planning. Our Panel's expansion to cover anal cancer parallels the expanding scope of that disease as well with increased rates of HPV infection. Several of our members are involved on a national and international level with research, education, and medical practice policy which positioned us well to author the articles in this issue. We would like to thank the Editor-in-Chief Dr. Johnson and the publisher Springer for this extraordinary opportunity.

In this issue, we include a systematic overview on topics from cancer of the colon to cancer of the anal canal. The two introductory articles discuss differences in imaging recommendations between the European Guidelines (ESGAR) and the American Guidelines (SAR), followed by a lexicon that will be useful in advance of reading the other articles in this Special Issue. There are perspectives written by other key physician members of the multidisciplinary care team on the importance of pathologic specimens and on the growing role of genetics in rectal cancer diagnosis and treatment. The burgeoning field of artificial intelligence, radiomics, and radiogenomics is also covered. Finally, several original research articles relevant to rectal cancer, some authored by non-DFP members, are also included to round out what we hope will prove to be a highly educational, useful and well-illustrated resource for the readership of Abdominal Radiology.

Publisher's Note Springer Nature remains neutral with regard to jurisdictional claims in published maps and institutional affiliations.

Marc J. Gollub

gollubm@MSKCC.ORG

Mukesh Harisinghani

MHARISINGHANI@MGH.HARVARD.EDU

1 Department of Radiology, Memorial Sloan Kettering Cancer Center, New York, USA

2 Massachusetts General Hospital, Harvard Medical School, Boston, USA 